TM- 1309

둘 Fermilab

1700

USING THE TEVATRON BEAM POSITION MONITOR

SYSTEM TO INVESTIGATE TRANSVERSE PHASE SPACE

F. Willeke

May 1985 


\title{
Using the TEVATRON Beam Position Monitor System to Examine Transverse Phase Space
}

\author{
F. Willeke
}

April 1985

\begin{abstract}
Transverse phase space trajectories can be measured by using the TEVATRON beam position monitor in the standard turn by turn operation mode if a coherent betatron oscillation is excited by a dipole kick. The measurement of phase space coordinates is influenced by incoherent and coherent synchrotron oscillations and incoherent betatron oscillations. In order to investigate phase space trajectories in detail, all these effects must be understood quantitatively and if possible the measured data must be corrected. In this report the measuring and correction procedures will be investigated and the first measurements will be discussed.
\end{abstract}

\section{Introduction}

The TEVATRON beam position monitor system (BPM) $/ 1,2,3 /$ is a powerful tool for beam diagnostics. In the Turn By Turn (TbT) mode, it allows one to measure the orbit of a bunch performing coherent betatron oscillations on 1024 successive turns at a single position monitor station. Such measurements provide not only information on coherent betatron oscillations but also on coherent and incoherent synchrotron oscillations, incoherent betatron oscillations and the strength and phase of linear coupling between the two transverse oscillation modes.

It is also possible to combine the data of two next neighbouring monitors to obtain the phase trajectory of a coherent transverse motions which helps to understand the behaviour of the machine under normal running conditions and allows investigation of the machine under special experimental conditions in order to test predictions of machine calculations. In particular there is large interest in the impact of nonlinear fields on the motion of the beam and how well it is described by the models used. Phase space measurements are therefore part of the effort to improve and optimize the calculational tools for designing new machines.

Because of the complexity of the motion under the influence of nonlinear fields, phase space distortions have to be measured with a great accuracy in order to be useful in the sense mentioned above. It is therefore necessary to have a quantitative understanding of all the effects which influence the data from such 
measurements, to isolate the nonlinear aspects of motion one is interested in.

We will start with a description of the experimental set up and will first discuss the role of chromaticity correcting sextupole fields and nonlinear components of the dipole fields between the two position monitors on the measurement of the slope of a betatron trajectory.

The coherent betatron amplitude as seen by TbT monitors is modulated, damped and dephased by the effect of incoherent motion within the particle bunch.

First we examine the impact of coherent and incoherent synchrotron motion. As we will see, the incoherent synchrotron motion results in a beat of the coherent betatron amplitude if the chromaticity is not zero.

Then incoherent betatron oscillations will be considered. If the particle tunes depend on the betatron amplitudes, the incoherent betatron motion gives rise to a relaxation of coherency which appears like a damping of coherent motion.

Finally we consider the effect of linear coupling. To study basic problems it may be sensible to study the motion in one oscillation plane only. Spurious linear coupling will disurb this motion and and has to be taken into account for interpretation of the data.

Having prepared our tool we will be able to discuss some of the measurements from the experimental program.

\section{Measuring Phase Space Trafectories Using} Turn by Turn Data

The experimental set up for the phase space measurements is the standart turn by turn operation mode of the BPM /1/: Two of the 24 service houses around the TEVATRON where the analog data from the 231 BP monitors get processed and digitized are equipped with a special processor which provides the turn by turn data. The TbT monitors can be chosen anywhere within the E2 or E4 section of the TEVATRON and may be horizontal or vertical or mixed. Suppose a coherent betatron oscillation is excited by a kick. This oscillation can be observed by the TbT monitors. We focus on the data of two next neighbouring monitors in the same plane and easily calculate the slope $z^{\prime}$ from the two position measurements $z \quad(z=x, y)$ at the two detectors (denoted by ' $i$ ' and ' $f$ ') by the linear transformation: 


$$
\begin{aligned}
{\left[\begin{array}{l}
z_{z^{\prime}} \\
z_{\text {final }}
\end{array} \quad T\left[\begin{array}{l}
z^{\prime} \\
z^{\prime}
\end{array}\right]_{\text {initial }}\right.} & z_{i}^{\prime}=\frac{z_{f}-T_{11} \cdot z_{i}}{T_{12}} \\
T= & {\left[\begin{array}{ll}
\left(\begin{array}{l}
\beta_{f} \\
\bar{\beta}
\end{array}\right)^{1 / 2}\left(\cos (\Delta \Phi)+\alpha_{i} \sin (\Delta \Phi)\right) & \left(\beta_{f} \beta_{i}\right)^{1 / 2} \sin (\Delta \Phi) \\
\frac{\left(\alpha_{f}-\alpha_{i}\right) \cos (\Delta \Phi)-\left(1+\alpha_{f} \alpha_{i}\right) \sin (\Delta \Phi)}{\left(\beta_{f} \beta_{i}\right)^{1 / 2}}\left(\begin{array}{l}
\beta_{i} \\
\bar{\beta}_{f}
\end{array}\right)^{1 / 2}\left(\cos (\Delta \Phi)-\alpha_{f} \sin (\Delta \Phi)\right)
\end{array}\right] }
\end{aligned}
$$

However this is only correct for very small oscillations for which the nonlinear forces between two monitors acting on the bunch are negligible. For large amplitudes the nonlinear fields may contribute to the transformation from one detector to the next one. The linear transformation has to be replaced by a nonlinear one which reads in the thin lens representation

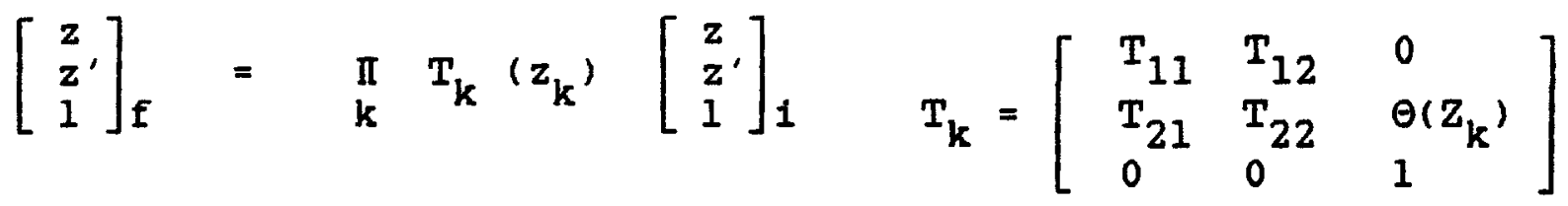

Here $\theta$ is the nonlinear kick in the middle of a sextupole or a dipole magnet:

$$
\Theta=\sum_{n, m} b_{n m} \cdot x^{n} y^{m} ; \quad b_{n m}: \text { multipole coefficients }
$$

Then the transformation equation cannot be solved for $z$ ' in a closed form (in general) and has to be treated iteratively. Starting with $\Theta=0$, thus applying the linear transformation, the nonlinear kicks for the next iteration loop can be calculated. In this step, by applying the quasi linear transformation

$$
\left[\begin{array}{l}
z \\
z^{\prime} \\
1
\end{array}\right]_{f}^{(2)}=\prod_{k} T_{k}^{(1)}\left[\begin{array}{l}
z \\
z \\
1
\end{array}\right]_{1}^{(2)}
$$

the nonlinear kicks for the following step are calculated and so on. Assuming the average of measured multipoles /4/ in each of the 8 dipoles and taking the amount necessary for chromaticity correction for the 2 standard sextupoles between two horizontal detectors, the nonlinear transformation differs from the linear one by $8 \%$ if the amplitude is as large as $15 \mathrm{~mm}$ which is about the maximum allowed one in the TEVATRON (quench protection). Because the main contribution arises from the sextupoles (70\%) the effect scales with the square of the amplitude and is therefore for amplitudes 
below $8 \mathrm{~mm}$ within the resolution limit of the detector system (which is $\simeq 0.2 \mathrm{~mm}$ )

However it should be mentioned that the nonlinear procedure is absolutely necessary for obtaining phase space trajectories at very low energies - at energies below $100 \mathrm{GeV}$ (e.g. at deceleration experiments). Here with an oscillation amplitude of only $5 \mathrm{~mm}$, the contribution of the persistent current sextupole components of the dipoles to $z^{\prime}$ is almost $20 \%$.

At this point I want to include a remark about averaging the data to remove the closed orbit distortion at the detectors and to center the phase space plots derived from turn by turn data. It is clear that with strong nonlinear contributions to the transformation from detector to detector, both the averaged data and the unaveraged data have to be transformed resulting in a $z^{\circ}$ and $z^{\circ}$ for the closed orbit and $z, z^{\prime}$ for unaveraged data. Phase space plots may then be produced from $\left(z-z^{\circ}\right),\left(z^{\prime}-z^{\circ}\right)$. It is not quite obvious that averaging always results in the closed orbit $z^{\circ}$ independently from what the phase space distortion is. It can however easily be demonstrated:

Suppose the canonical variables are the particle betatron phase $\Phi$ and the particle emittance $\varepsilon$, both invariants if all forces are linear. Thus phase space trajectories $\varepsilon(\Phi)$ (which correspond to $\left(X^{\prime}=x \alpha+x^{\prime} \beta\right.$ over $\left.x^{\prime}\right)$ ) are circles enclosing an area of $2 \pi \varepsilon^{\circ}$. Suppose the nonlinear distortions are turned on adiabatically. Then the phase space trafectories get distorted and $\varepsilon$ becomes a complicated function of $\Phi$. However because the time development of $\varepsilon(\Phi)$ can be considered as a canonical transformation, the poincare integral $\int d \Phi \varepsilon(\Phi)$ is invariant, thus $\int d \Phi \varepsilon(\Phi)=2 \pi \varepsilon^{\circ}$. The general description of of a phase space distortion which conserves the phase space area is a Fourier expansion of $\varepsilon$ about $\varepsilon^{\circ}$ in $\Phi$ :

$$
\varepsilon(\Phi)=\varepsilon^{0}\left(1+\Sigma_{n=1}^{\infty} a_{n}\left(\varepsilon^{0}\right) \cos \left(n \Phi+\Phi_{n}\right)\right)
$$

The coordinate $z$ on successive turns can be written now as

$$
\begin{aligned}
& z(n)=z^{0}+\left(2 \varepsilon^{\circ} \beta_{z}\right)^{1 / 2} \\
& \text { - }\left(\cos (\Phi)+\frac{1}{4} \Sigma_{n} a_{n}\left(\cos \left((n+1) \Phi+\Phi_{n}\right)+\cos \left((n-1) \Phi+\Phi_{n}\right)\right)\right. \\
& -\frac{1}{128} \sum_{n, n^{\prime}} a_{n} a_{n^{\prime}}\left(\cos \left(\left(n+n^{\prime}+1\right) \Phi+\Phi_{n}+\Phi_{n^{\prime}}\right)+\cos \left(\left(n-n^{\prime}+1\right) \Phi+\Phi_{n}-\Phi_{n^{\prime}}\right)\right) \\
& +\Sigma_{n, n^{\prime}, n^{\prime}}
\end{aligned}
$$

If all parts on the phase space trajectory are occupied with the same density by measured phase space coordinates, averaging over all $z^{\prime}$ s thus averaging over all phases $\Phi$ always results in the closed orbit deviation $z^{\circ}$ no matter how complex or how large the distortion or what the phase of the distortion will be. 


\section{Influence of Synchrotron Oscillations on Turn by Turn Data}

In this section we will discuss the impact of coherent and incoherent synchrotron oscillations on a coherent B-tron oscillation.

A coherent synchrotron oscillation will be visible on turn by turn data if the dispersion at the turn by turn monitor is not zero. Assuming a harmonic coherent synchrotron oscillation the TbT data are described by :

$$
x(n)=x^{0}+D_{x_{s}}^{c} \cos \left(2 \pi Q_{s}^{c} n+\phi_{s}^{c}\right)
$$

with

$Q_{s}^{C}, \varepsilon_{s}^{C}, \phi_{s}^{C}$ coherent synchrotron tune, amplitude, phase

$D_{x}$ value of the dispersion function at the monitor

Such oscillations are observed very often at injection as a consequence of momentum or phase errors of the injected beam or a detuning of the $r f$ frequency. If there is a momentum error, the oscillation observed on TbT is cosine-like and sinelike for a phase or frequency error. Injection phase errors excite sine like synchrotron oscillations. A momentum error causes a coslike synchtrotron oscillations whereas an rf frequency error has the form $1-\cos (2 \pi, n)$. Momentum exrors and rf frequency errors can only be distinguished knowing the on energy closed orbit deviation at the TbT detector. Fig 1 shows a TbT measurement made at TEVATRON injection. The synchrotron oscillation was exited in this case by a momentum error.

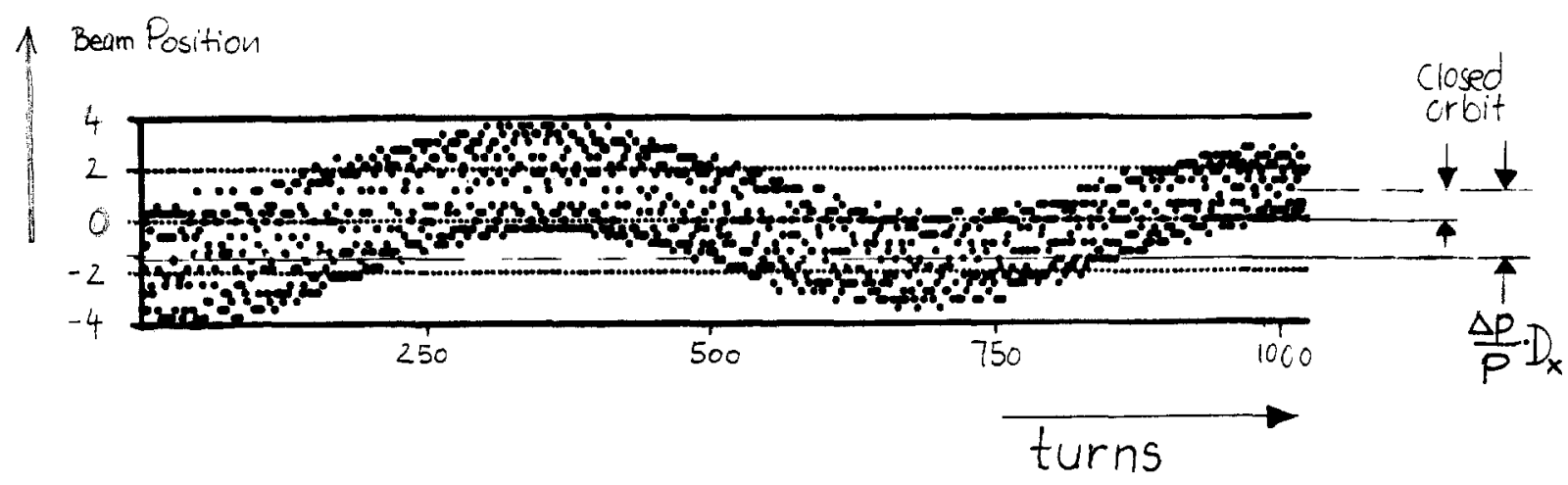

fig 1. Example for a Coherent Synchrotron 0scillation as Seen by the Turn by Turn Monitor

An incoherent sychrotron oscillation leads to a dilution of the coherent betatron oscillation. However after one synchrotron cycle coherency gets restored. The phenomenon can be described in a closed form assuming a gaussian energy spread in the bunch and 
assuming the incoherent oscillation to be harmonic. The amplitude of a single particle in a bunch performing a coherent betatron oscillation about the closed orbit may then be written as

$$
x(n)=x_{0} \cos \left(2 \pi Q_{x} n+\Phi_{x}+2 \pi \cdot \Sigma_{k=1}^{n} \Delta Q_{k}\right)
$$

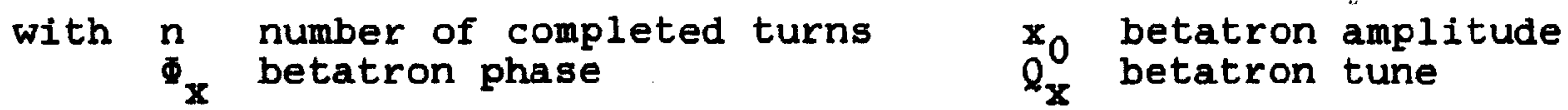

$\Delta Q_{k}$ tune change due to momentum error

$$
\begin{aligned}
& \Delta Q_{k}=\xi \cdot \varepsilon \cdot \sin \left(2 \pi Q_{s} \cdot k+\phi_{s}\right) \\
& \sum_{k=1}^{n} \Delta Q_{k}=\xi \cdot \varepsilon \cdot \sin \left(\pi Q_{s} n\right) \cdot \cos \left(\pi Q_{s}(n+1)+\phi_{s}\right) / \sin \left(\pi Q_{s}\right)
\end{aligned}
$$

with $Q_{\mathbf{s}}, \Phi_{\mathbf{s}}, \varepsilon$ incoherent synchrotron tune, phase and amplitude $\xi \quad$ chromaticity $\Delta Q /(\Delta p / p)$

Different particles differ by their incoherent synchrotron amplitude and phase. Because the beam position monitor essentially accumulates contributions of different particles, the observable coherent oscillation is an integral over all momenta and phases:

$$
\overline{x(n)}=\int_{-\infty}^{+\infty} d \varepsilon \int_{0}^{2 \pi} d \phi \quad x(n) \cdot \rho(\varepsilon, \phi), \rho: \text { distribution function }
$$

If the momentum distribution is gaussian and the phases are distributed uniformly, the momentum averaging can be evaluated in a closed form:

$$
\begin{aligned}
& \overline{x(n)}=x^{\circ}(n) \cdot \frac{1}{2 \pi} \int d \Phi e^{\left.-\frac{1}{2}\left(\frac{2 \pi \xi \varepsilon^{\circ} \sin \left(\pi Q_{s}\right.}{\sqrt{2} \sin \left(\pi Q_{s}\right.}\right)\right)^{2} \cos ^{2}\left(\pi Q_{s}(n+1)+\phi_{s}\right)} \\
& x^{\circ}(n)=x^{\circ} \cdot \cos \left(2 \pi Q_{x}+\Phi_{x}\right), \text { undistorted } \beta \text {-tron oscillation }
\end{aligned}
$$

Here $\varepsilon^{0}$ is the rms value of the momentum spread. Remains to carry out the phase averaging. The cos-term in the exponent can be expanded about $1 / 2$ leading to a power series in

$\alpha=2 \pi \xi \varepsilon^{\circ} \cdot \sin \left(\pi Q_{s} n\right) / \sqrt{2} \cdot \sin \left(\pi Q_{s}\right):$

$$
\overline{x(n)}=x(n) \cdot e^{-\frac{1}{2} \alpha^{2}} \cdot\left[\Sigma_{n, m} \frac{(-1)^{n+m}}{n !}\left(\begin{array}{l}
n \\
m
\end{array}\right)\left(\begin{array}{c}
2 n-2 m \\
n-m
\end{array}\right)\left(\frac{1}{2}\right)^{2 n-m} \cdot \alpha^{2 n}\right]
$$

The sum over $n$ extends over even $n$ only; the sum over $m$ extends from $m=0$ to $m=n$. In lowest orders one obtains for the expansion: 


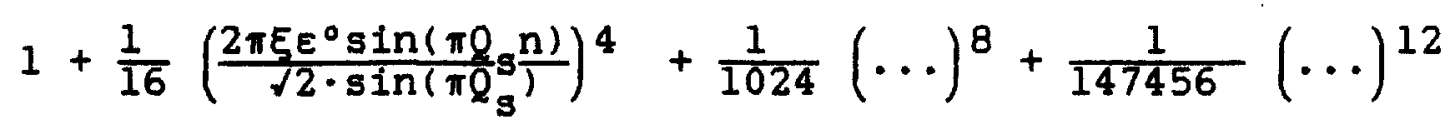

For proton beams, a gaussian distribution of the momentum spread may not be appropriate. A parabolic distribution is probably more realistic:

$$
\rho(\varepsilon)=\frac{4}{3 \varepsilon^{0}}\left(1-\left(\frac{\varepsilon}{\varepsilon_{0}}\right)^{2}\right)
$$

In this case the betatron amplitude is given by:

$$
\begin{aligned}
& \overline{x(n)}=x(n) \frac{1}{2 \pi} \int_{0}^{2 \pi} \mathrm{d} \phi 3\left(\sin (\tilde{\alpha}), \tilde{\alpha}^{3}-\cos (\tilde{\alpha}), \tilde{\alpha}^{2}\right) \\
& \tilde{\alpha}=2 \pi \xi \varepsilon^{\circ} \sin \left(\pi Q_{s} n\right) \cdot \cos \left(\pi Q_{s}(n+1)+\phi_{s}\right) / \sin \left(\pi Q_{s}\right)
\end{aligned}
$$

in lowest order one obtains

$$
\overline{x(n)}=x(n) \cdot\left(1-\left(\frac{2 \pi \xi \varepsilon^{\circ} \sin (\pi Q}{\sin \left(\pi Q_{g} \frac{n}{)}\right)}\right)^{2}+\ldots\right)
$$

In both cases, the effect of an incoherent synchrotron oscillation on the coherent betatron oscillation is a beat of the coherent amplitude with the incoherent synchrotron frequency. At half the synchrotron period the beat envelope has its minimum. The beat envelope is symmetric about this point. After one full synchrotron period the initial coherent amplitude gets completely restored. For the two distributions we considered, the amplitude beat factors differ numerically only little.

Fig 2 shows the beat factor for a gaussian distribution in longitudinal phase space. The plot extends over a full synchrotron period. The parameter $\alpha$ of the curve is a measure of the beat factor, $\alpha=2 \pi \cdot \xi \varepsilon^{\circ} / \sqrt{ } \cdot \sin \left(\pi Q_{s}\right)$. Up to $\alpha \simeq 0.5$, the beat is described very well by the zero-th order term

$$
\text { beat } \sim e^{-0.5 \alpha^{2}}
$$

If $\alpha$ gets as large as 1.5, the 2nd order term in the expansion contributes with $31.6 \%$ and the 4 th order with still $2.5 \%$. For such $\alpha$ the bottom of the beat envelope flattens and for very large values eventually forms a local maximum at half the synchrotron period 


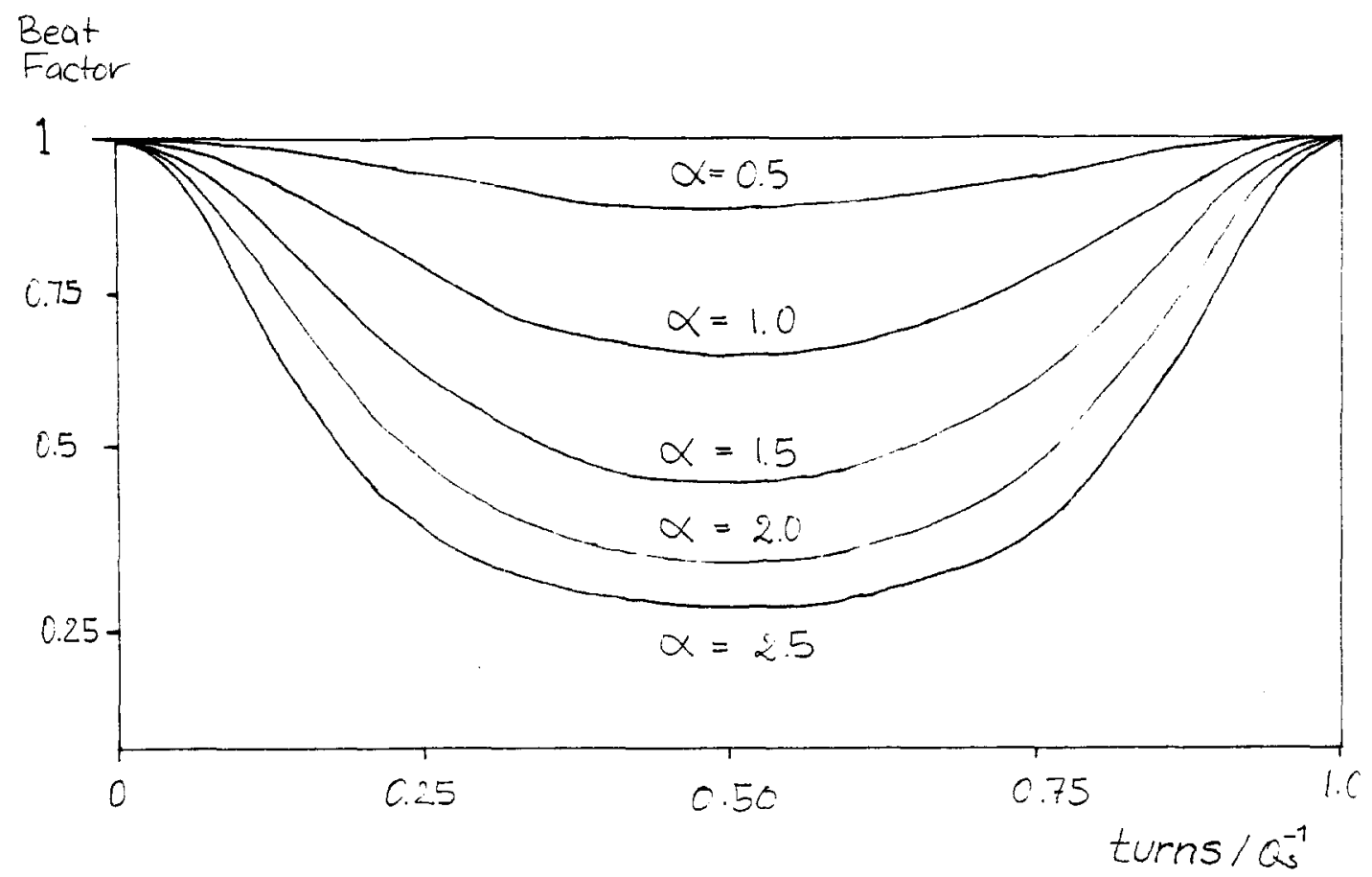

fig 2 Beat Factor Due to Incoherent Synchrotron Oscillations for Different Beat Strengths $\alpha=2 \pi \varepsilon^{\circ} \xi / \sqrt{2} \cdot \sin (\pi Q)$ as a Function of Turns

The above formula describe very well what is observed in turn by turn measurements. Fig 3 shows two examples of a coherent betatron oscillation influenced by incoherent momentum oscillations, which differ by their momentum spread by a factor of about 3 . Note the small local minimum of the beta factor when beating is strong. 


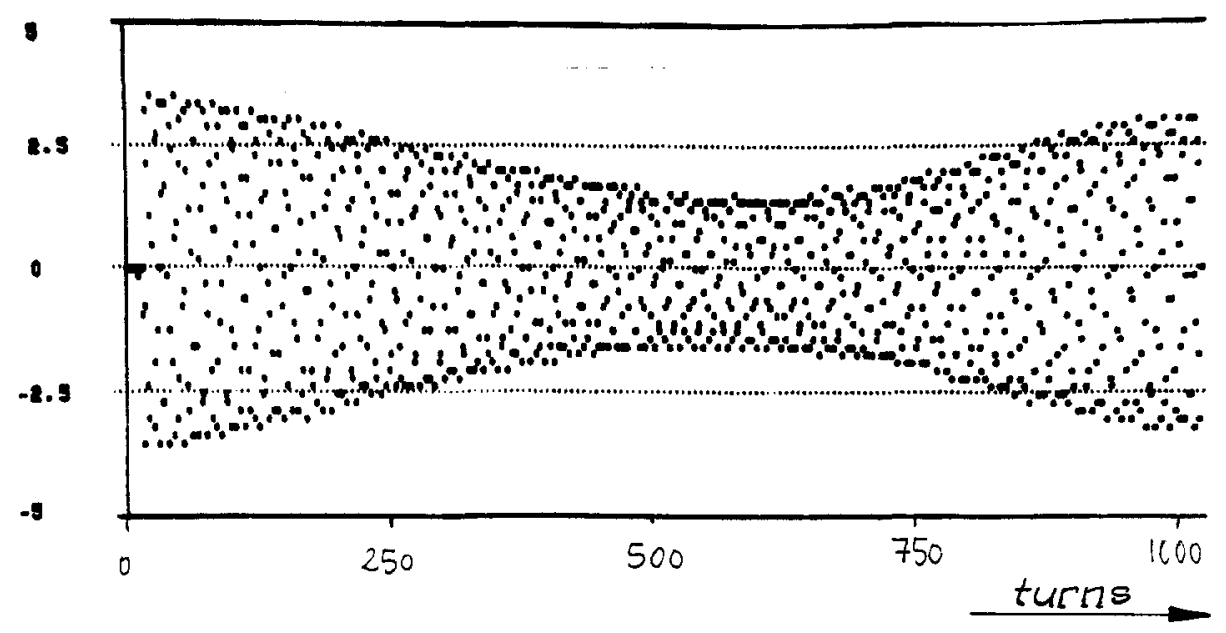

fig 3a Example of Turn by Turn Data with Moderate Beat Factor Due to Incoherent Synchrotron Oscillations

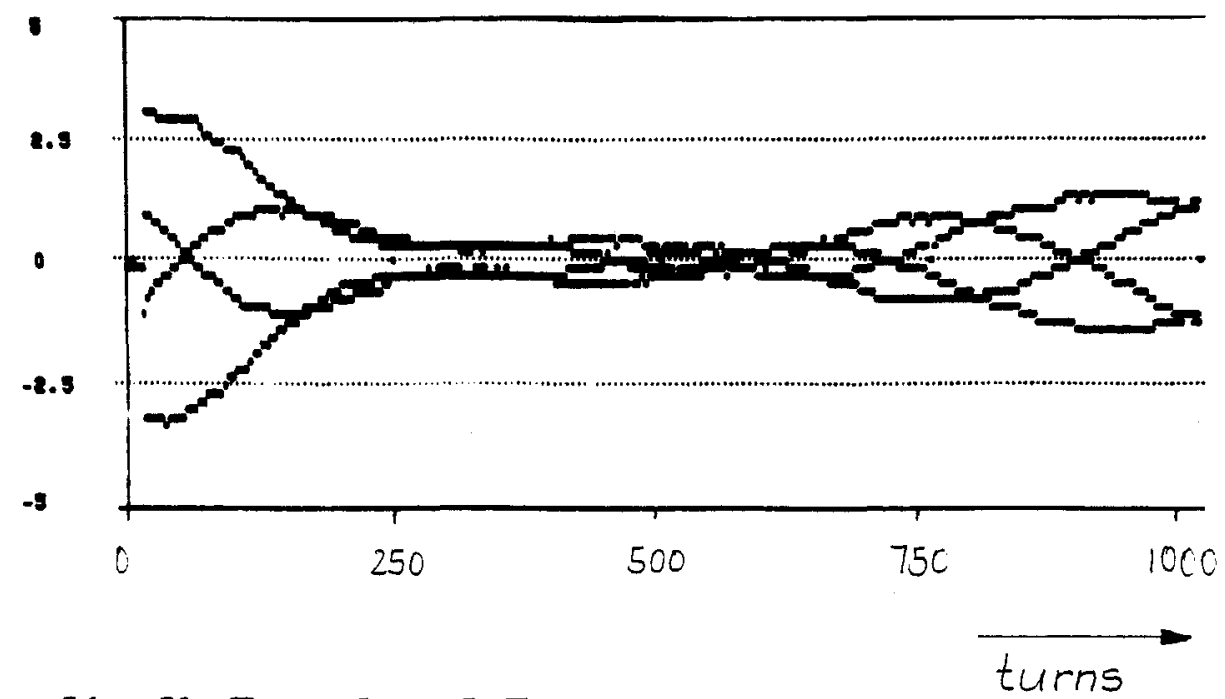

fig 3b Example of Turn by Turn Measurements with strong Synchrotron Beat Factor

If no other perturbations are present, it should be possible to evaluate two important machine parameters from turn by turn measurements 
* the incoherent momentum spread in the beam

t the incoherent synchrotron frequency, providing information about collective longitudinal self forces in a bunch

\section{Influence of Incoherent Betatron Oscillations}

If the betatron tune depends on the oscillation amplitude, one observes a damping of the coherent amplitudes. Such amplitude dependent tunes may be caused by transverse inner bunch forces (e.g. space charge effects ). However at TEVATRON energies, the only important contributions come from nonlinear fields around the machine. Far away from a resonance, the so called detuning terms are produced by the average value of even ordered multipole components ( octupoles, 12-poles etc) or higher order contributions from odd ordered multipoles (sextupoles, decapoles,...) in the machine. The general form of the amplitude dependent tune is:

with

$$
\Delta Q_{x}=\beta_{x} \frac{\partial}{\partial\left(x^{2}\right)} \sum_{n, m}^{\text {even }} \quad a_{n m}\left(\frac{x^{2}}{\bar{\beta}_{x}}\right)^{n / 2}\left(\frac{y^{2}}{\beta_{y}}\right)^{m / 2}
$$

$$
\beta_{x}, \beta_{y} \text { beta functions } a_{n m} \text { 'detuning' terms }
$$

Because we cannot operate with extreme amplitudes when taking phase space plots, in practice only the lowest order terms are important in the expansion and we will take into account only terms proportional to $x^{2}$ and $y$. Assuming that the particles are distributed gaussian in the bunch, the average value of the beam position over all individual particles characterized by oscillation amplitudes $\left(x+x^{0}\right),\left(y+y^{0}\right) \quad\left(x^{0}, y^{\circ}\right.$ being the coherent amplitudes) can be written as the integral:

$$
\begin{array}{r}
\overline{x(n)}=\frac{2 x^{0}}{\pi \sigma_{x} \sigma_{y}} \int_{-\infty}^{+\infty} d x \int_{-\infty}^{+\infty} d y \cdot\left(\cos \left[2 \pi n \cdot\left\{Q_{x}+A_{x}\left(x+x^{0}\right)^{2}+A_{y}\left(y+y^{\circ}\right)^{2}\right\}+\Phi_{x}\right]\right. \\
\left.\cdot e^{-x^{2} / 2 \sigma_{x}^{2}} \cdot e^{-y^{2} / 2 \sigma_{y}^{2}}\right)
\end{array}
$$

This expression can be evaluated resulting in 
$\overline{x(n)}=x^{\circ} \cos \left[2 \pi n\left(Q_{x}+\Delta Q_{x}\right)+\Phi_{x}+\Phi(n)\right] \frac{e^{-\frac{1}{2} \frac{\left(4 \pi n A_{x} \sigma_{x} x^{\circ}\right)^{2}}{1+\left(4 \pi n A_{x} \sigma_{x}^{2}\right)^{2}}-\frac{1}{2} \frac{(4 \pi n A}{\left.1+\left(4 \pi n A_{y} \sigma^{\circ}\right)^{2}\right)^{2}}}}{\left(1+\left(4 \pi n A_{x} \sigma_{x}^{2}\right)^{2}\right)^{1 / 4}\left(1+\left(4 \pi n A_{y} \sigma_{y}^{2}\right)^{2}\right)^{1 / 4}}$

here

$\Delta Q_{X}$ the tune change according to mean amplitudes $x^{\circ}, y^{\circ}$ $\Delta Q_{x}=A_{x} x^{02}+A_{y} y^{\circ 2}$

$\Phi(n)=\frac{1}{2}\left(\tan ^{-1}\left(\eta_{x}\right)+\tan ^{-1}\left(\eta_{y}\right)+\frac{\zeta_{x} n_{x}}{1+\eta_{x}^{2}}+\frac{\zeta_{y} \eta_{y}}{1+\eta_{y}^{2}}\right)$

with $\eta_{x}=4 \pi A_{x} n \sigma_{x}^{2}, \zeta_{x}=4 \pi A_{x} n \sigma_{x} x^{0}$, ditto for $y$

$\sigma_{x} \sigma_{y}$ are horizontal and vertical rms beam sizes

$A_{x}=2 a_{20} / B_{x} \quad A_{y}=a_{11} / B_{y} \quad \begin{aligned} & \text { are the lowest order } \\ & \text { detuning terms }\end{aligned}$

These expressions show that the influence of a tune spread is essentialiy a damping of the coherent amplitude driven by the detuning terms and the beam size. There is also a change in the coherent betatron phase $\Phi(n)$ because the defuning for $x>x^{\circ}$ is larger than for $x<x^{\circ}$. This is due to the $x^{2}$ dependence of the tune spread, thus the phase spread is nonsymmetric. It is necessary to correct for this phase error on the phase space plot unless the phase space trajectories are circles. One should notice that the impact of horizontal and vertical beam size on the damping factorizes into a horizontal and vertical contribution. It is also clear that the effect of sychrotron oscillations as described in the preceding section also just contributes by another factor, the beat factor described above. In fig 4 the damping factor is plotted over the number of completed turn for a case where the coherent amplitude $x^{\circ}$ is the same as the beam size $\sigma$ ( which corresponds to a $4 \mathrm{~mm}$ betatron oscillation at TEVATRON injection). Fig 5 shows turn by turn data influenced by amplitude dependent tune spread. 


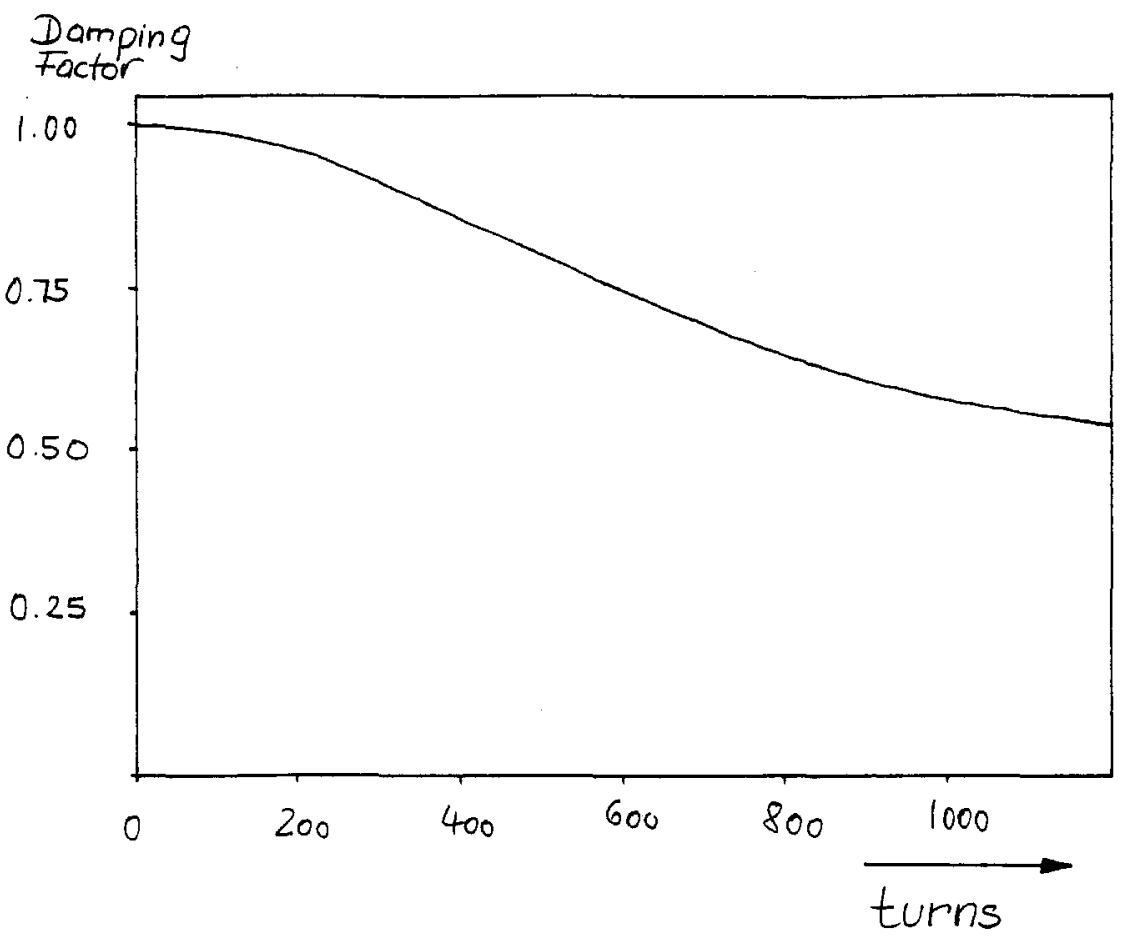

fig 4 Form of the Damping Factor for $x^{\circ}=\sigma$ and a Detuning of $\Delta Q_{x}=0.0001$ at $x=\sigma_{x}^{x}$

beamposition $/ \mathrm{mm}$

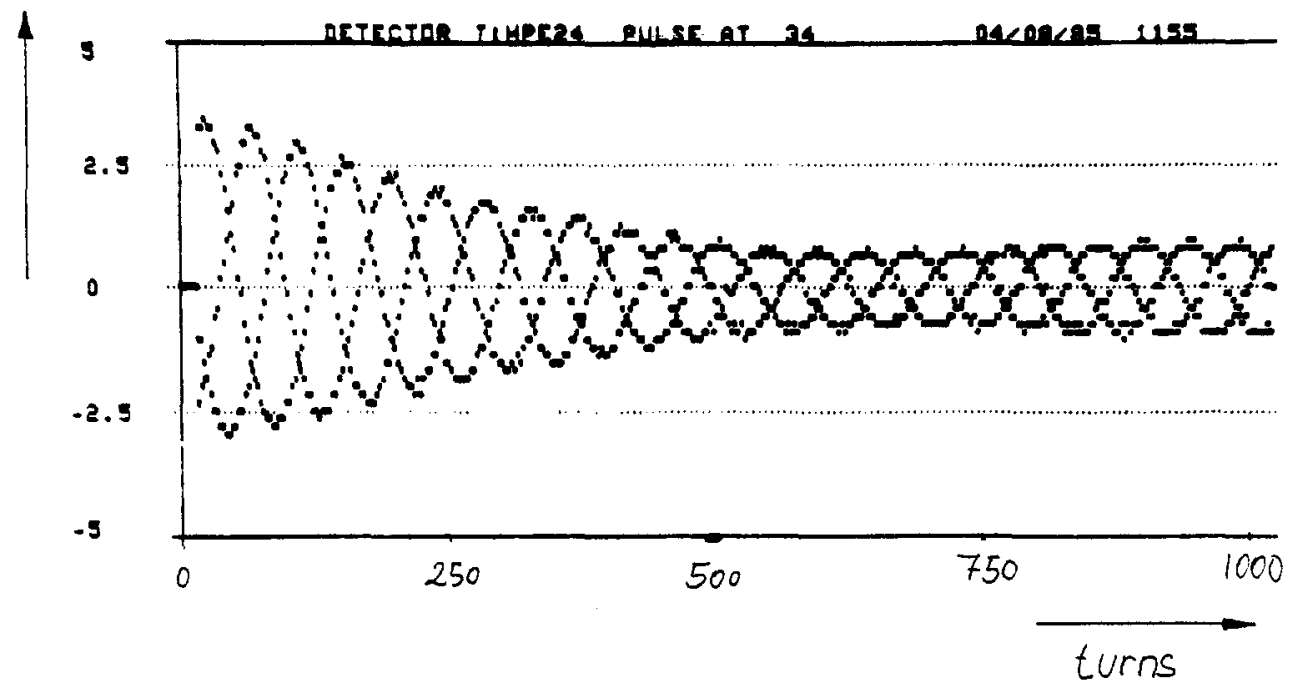

fig 5 Turn by Turn Measurement near the 3rd Integer Resonance with Damping of the Coherent Amplitude Due to Detuning Terms 
This form of the damping allows in principle the evaluation of the beam radius $\sigma^{2}+\sigma^{2}$ if the detuning terms are known. One could think of a strong zero harmonic octupole excited for that purpose. In the same experiment it would be possible to measure the detuning terms by varying the coherent amplitude and measuring the betatron tune of the coherent oscillation.

\section{Influence of Linear Coupling}

The presence of linear coupling makes it difficult to investigate phase space trajectories in one oscillation plane. It is therefore desirable to study the particle motion in the eigenmodes of the coupled system rather than in the $x$ and $y$ plane. For the sake of completeness the transformation between eigenmodes I,II and the machine coordinate system $x, y$ will be listed below according to reference/5/

In the case of linear coupling it is possible to preserve the familiar lattice functions $\beta, \alpha, \gamma$ and like in the uncoupled case. However for both $x$ and $y$ plane we have to add $a$ second mode which vanishes for zero coupling :

$$
\begin{aligned}
& x=\sqrt{\varepsilon_{I} \beta_{X I}} \cdot \cos \left(\Phi_{X I}+\Phi_{I}\right)+\sqrt{\varepsilon_{I I} \beta_{X I I}} \cdot \cos \left(\Phi_{X I I}+\Phi_{I I}\right) \\
& x^{\prime}=\sqrt{\varepsilon_{I} \gamma_{X I}} \cdot \cos \left(\Phi_{X I}+\tilde{\Phi}_{I}\right)+\sqrt{\varepsilon_{I I} \gamma_{X I I}} \cdot \cos \left(\Phi_{X I I}+\tilde{\Phi}_{I I}\right) \\
& y=\sqrt{\varepsilon_{I} \beta_{y I}} \cdot \cos \left(\Phi_{y I}+\Phi_{I}\right)+\sqrt{\varepsilon_{I I} \beta_{Y I I}} \cdot \cos \left(\Phi_{Y I I}+\Phi_{I I}\right) \\
& \mathrm{Y}^{\prime}=\sqrt{\varepsilon_{I} \gamma_{\mathrm{Y} I}} \cdot \cos \left(\Phi_{\mathrm{Y} I}+\tilde{\Phi}_{I}\right)+\sqrt{\varepsilon_{I I} \gamma_{\mathrm{Y} I I}} \cdot \cos \left(\Phi_{\mathrm{Y} I I}+\tilde{\Phi}_{I I}\right)
\end{aligned}
$$

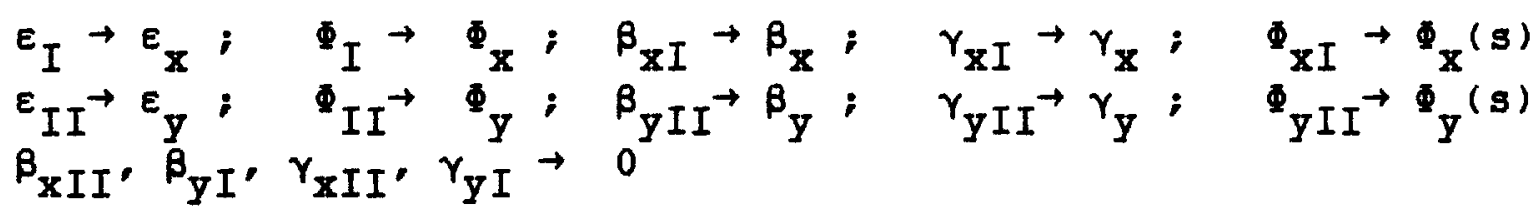

Because the BPM system allows retrieval of the TbT data of all the horizontal and vertical monitors at the same time, it is straight forward to obtain the lattice functions and invariants for both modes from a fit to $x(n)$ and $y(n)$. This enables us to study the two eigenmodes independently as far as linear coupling is concerned. 
If the phase space plot in one plane (e.g. $x$ ) is performed only for points with the same phase in the other plane ( $y$ ), the phase space plot results in an ellipse. The excentricity of the elilpse is a measure of the strength of the skew quadrupole representing the distortions around the ring. Near the coupling resonance $Q_{x}=Q_{y}$, the
ratio $R$ between maximum and average phase space radius

$R^{2}=\left(x^{2}+\left(x \cdot \alpha+x^{\prime} \beta\right)^{2}\right)_{\max } /\left(x^{2}+\left(x \alpha+x^{\prime} \beta\right)^{2}\right)$ average

is related to the strength of the skew quadrupole $\mathrm{kl}_{\text {skew }}$ by

$$
R^{2}=\left(\left(\beta_{x} \beta_{y} / 4\right)^{1 / 2} k 1_{\text {skewq }}\right)\left(\left(y^{\circ} / x^{0}\right)\left(\beta_{x} / \beta_{y}\right)^{1 / 2} \operatorname{detector}\right) / 2 \sin \left(\pi Q_{x}-\pi Q_{y}\right)
$$

The angle between the ellipse main axis and the $x$ axis is the betatron phase difference between the monitor and this skew quadrupole. The same can be done for the other plane which provides full information about the skew quadrupoles necessary to correct for linear coupling.

\section{Phase Space Measurements}

Some phase space measurements have been done so far which will be described as an example. These measurements are also described in the context of other TEVATRON experiments for SSC related questions in ref $/ 6 /$.

According to a propososal of Sho Ohnuma and Don Edwards /7/, 8 sextupoles around the ring are powered in two circuits such that they drive mostly 2 nd and $3 r d$ integer resonances $(2 Q, 3 Q=i n t e g e r)$ or by changing the sign of one of the circuits, they dive mainly lrst and 4 th integer resonances $\left(1,4 Q_{4}=\right.$ integer $)$.

Beam was injected in the machife and accelerated to $400 \mathrm{GeV}$. At flat top the sextupoles were switched on smoothly. The beam was then kicked in the horizontal plane applying a kick of $\simeq 40 \mu \mathrm{rad}$. Phase space trajectories were obtained by using the two horizontal beam position monitors at positions E22 and E24 or E24 and E26 respectively.

The original data were strongly influenced by both incoherent synchrotron and betatron oscillations. The tunes were well separated with $q_{\mathrm{X}} \simeq 19.33, .25, \mathrm{Q}_{\mathrm{Y}} \simeq 19.42$. Thus operating far from the couplifing resonance, Ilnear coupling could be suppressed sufficiently, so that it had no impact on the data.

Because the experiment was been performed at $400 \mathrm{GeV}$, the contribution of nonlinear flelds between the position monitors turned out to be negligible. Their contribution to the evaluation of 
the trajectory slopes have been found to be less than $0.2 \%$ using the measured multipole fields. This is much below the noise level seen on the phase space plots.

The data handling was as follows:

First an envelope function for each set of turn by turn by turn data has been determined by selecting the maxima and minima of the betatron oscillations. The beat and damping factors as derived above then have been fitted to the centered experimental envelope curve. Turn by turn data then get centered by subtracting the average value over all betatron oscillations and get scaled by the inverse fitted envelope function. Phase space points $x, x \alpha+x^{\prime} \beta$ can now be determined by applying the transformation described in the first section. Finally the phase space angle has to be corrected for the virtual phase change in the turn by turn data due to damping using the fitted values of damping parameters $\sigma_{x}, \sigma_{y}, A_{x}, A_{y}$ (see preceding sections).

Fig 6 shows an example corrected and uncorrected turn by turn data. The phase correction is not yet applied. The sextupoles have been powered to drive the 4 th integer resonance and the horizontal tune was adjusted to $Q_{x}=0.22$.

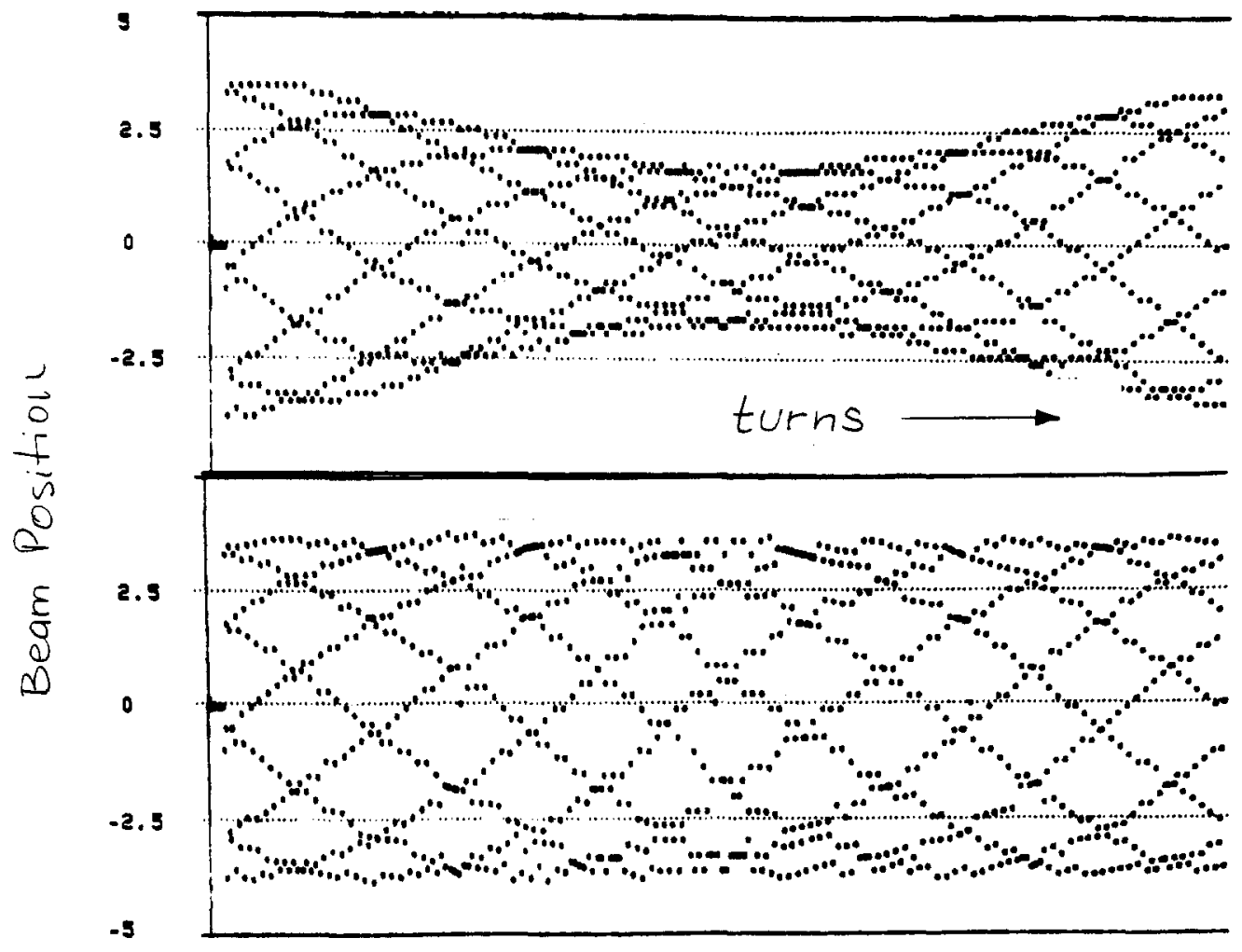

fig 6 Perturbed Turn by Turn Data and Appropriate Correction 
Phase space trajectories are almost perfect circles. Fig7 shows a comparison of phase space data from the corrected and uncorrected turn by data.

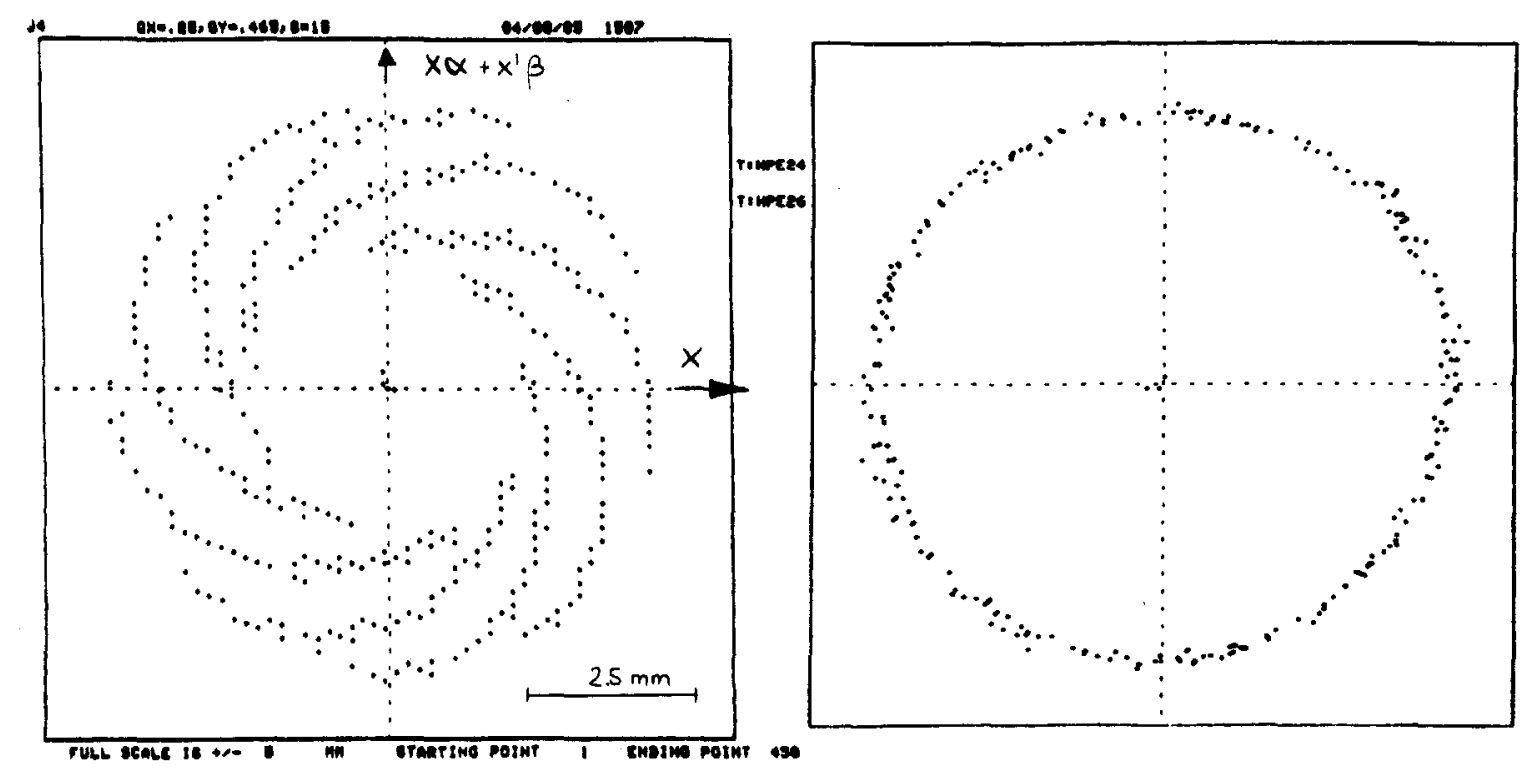

fig 7 Uncorrected and Corrected Phase Space Plots

When sextupoles are powered to drive the $3 \mathrm{rd}$ integer resonance, and the tune is moved near $\ell_{x} \simeq 19.33$ phase space trajectories are distorted into a triangular shape. Size and phase of the observed phase space distortion agree well with analytical estimates which has been made using perturbation theory / ref $8 /$ 


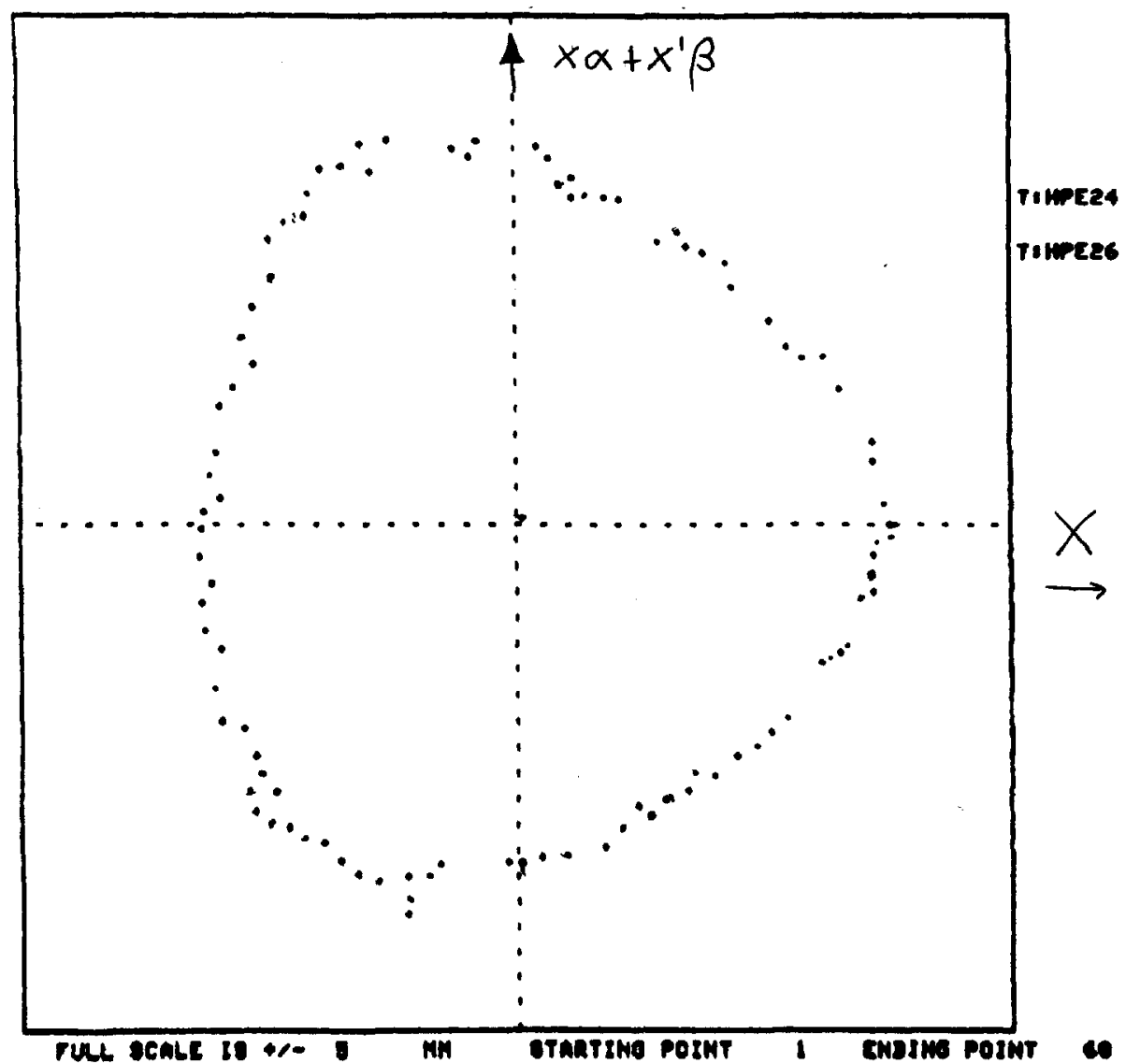

fig 8 Corrected Phase Space Plot near Third Integer Resonance

\section{Conclusion}

It has been demonstrated that turn by turn beam position monitor measurements can be used as a diagnostic tool to a much higher extent than it has been used up to now in the TEVATRON.

In particular it is possible to obtain detailed information about the distortions of transverse phase space trajectories caused by nonlinear fields and to check the validity of computer simulation or analytical models of particle motion in the accelerator.

\section{Acknowledgment}

I wish to thank Rod Gerig, Rol Johnson, Don Edwards and Leo Michelotti for discussions. 


\section{$\underline{\text { References }}$}

(1) R.E. Shafer and R.E. Gerig The TEVATRON Beam Position and Loss Monitoring System Proceedings of the XIth International Accelerator Conference 1983 Fermilab August 1983

(2) S.P.Jachim,R.C.Weber, R.EW.Shaf er An rf Beam Position Measurement Module for the Fermilab Energy Doubler IEEE Trans Nucl Sc,Vol NS-28 no3 june 1983

(3) R.Gerig, Fermilab Operations Bulletin \#888(fuly'82), no\#916(Sept'83), \#924(July'89)

(4) Norm Gelfand, Tevatron Data Base, not published

(5) G.Ripken, Untersuchung zur Strahlfuehrung and Stabilitaet der Tellchenbewegung in Beschleunigern und Storageringen unter strenger Beruecksichtigung einer Kopplung der Betatronschwingung DESY RI-70/4 June 1970

(6) Don Edwards, Rol Johnson and Ferdi Willeke, Test of Orbital Dynamics Using the TEVATRON Proceedings of the Workshop on Orbital Dynamics, Berkeley, march 1985

(7) Sho Ohnuma, Don Edwards, private communication

(8) Don Edwards, private communication 